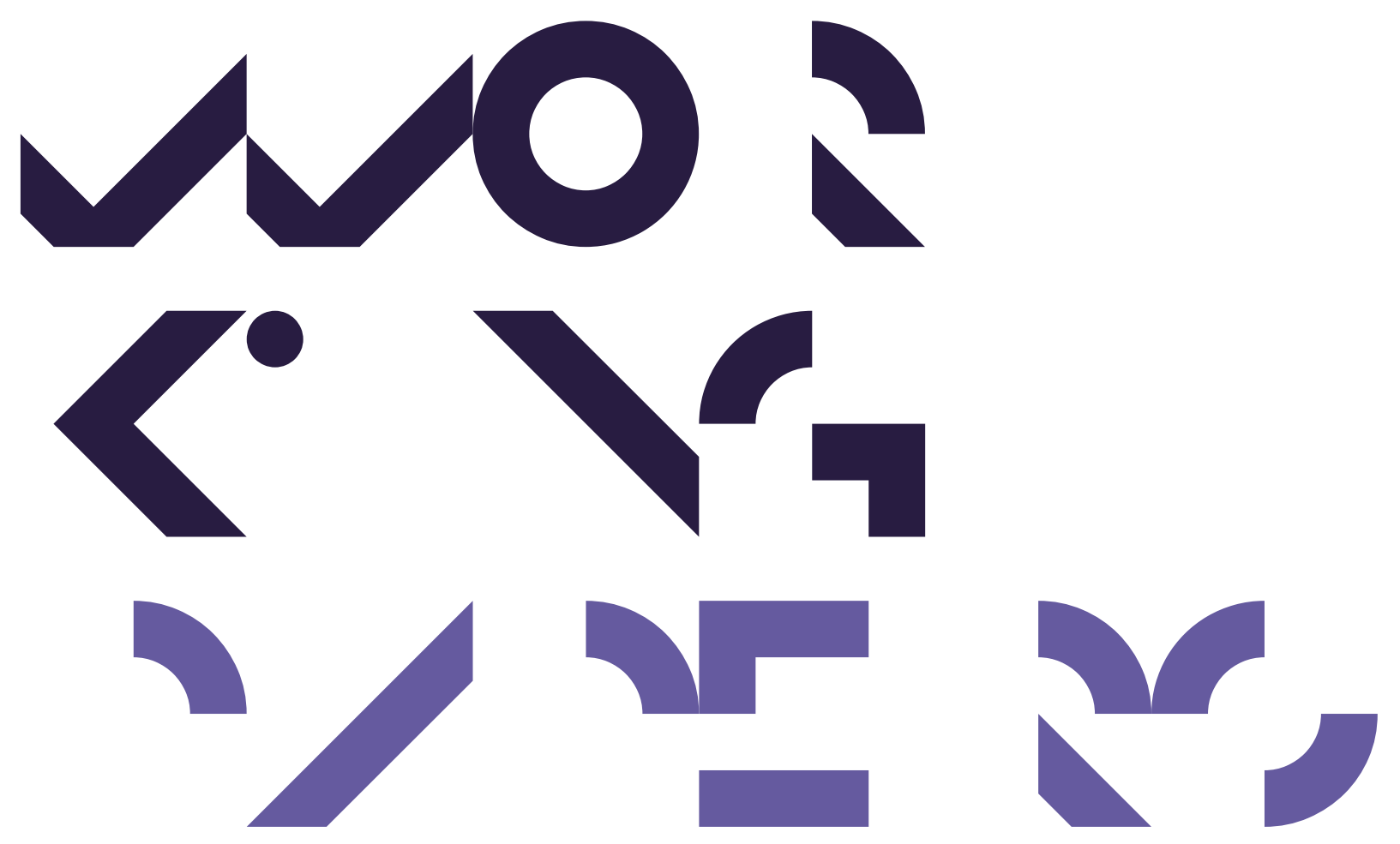

Economics Working Papers

2013-05

Determinants of Generic vs. Brand Drug Choice: Evidence from Population-wide Danish Data

Niels Skipper and Rune Vejlin 


\title{
Determinants of Generic vs. Brand Drug Choice: Evidence from Population-wide Danish Data
}

\author{
Niels Skipper ${ }^{\dagger}$, Rune Vejlin*
}

\begin{abstract}
:
When prescription medications go off patent, vastly cheaper generic drugs usually enters the market. However, the original brand medication often maintains non-negligible market shares. This paper investigates whether demand for branded medications in post-patent markets is patient- or doctor driven. We use population-wide Danish register data including all prescriptions for seven blockbuster drugs from 1998-2008. At the outset, descriptive statistics suggest large variation in drug choice over doctors. Nonetheless, using a two-way fixed effects model we find that the primary determinants of brand drug use are unobserved patient characteristics and price effects, while observed and unobserved doctor characteristics in general explain only $0.7 \%$ of the variation in drug choice. This is suggestive evidence that the doctors in the Danish setting with no incentives to push expensive brand drugs do indeed not do so. Our results also suggest that one should be careful when applying fixed effects in small samples.
\end{abstract}

JEL classification: I11, L65

Keywords: Prescription drug demand, fixed effects, brand preferences

Acknowledgements: We would like to thank Mariana Carrera for helpful comments and suggestions.

$\dagger$ Department of Economics and Business, Business and Social Sciences, Aarhus University, Fuglesangs Allé 4, 8210 Aarhus V., Denmark. Email: nskipper@econ.ku.dk.

* Department of Economics and Business, Business and Social Sciences, Aarhus University, Fuglesangs Allé 4, 8210 Aarhus V., Denmark. Email: rvejlin@econ.au.dk 


\section{Introduction}

Increasing prescription drug expenditures is an important policy issue in most developed countries. One of the contributors to elevated expenditures is brand-name drugs that are sold at high prices while the drug is under patent protection. As a consequence, third party payers (government and private) encourage generic substitution when these drugs go off-patent. Despite the fact that generic alternatives are often much cheaper than the original brand, brand-versions of a drug typically maintain a non-negligible market share after patent expiry, and little is known about the determinants of the choice between brand-name versus generics drugs.

The goal of this paper is to empirically assess the determinants of drug choice using populationwide register data from Denmark. We estimate a linear probability model for drug choice with two-way error components in the spirit of Abowd et al. (1999) that allows for identification of time-invariant unobserved heterogeneity on both the patient and doctor side simultaneously. This estimator has been widely used in the labor literature on matched employer-employee data. Due to data availability, few studies of doctor-patient interactions exists; see Koulayev et al. (2013) and Bennet et al. (2011). The estimation strategy is very demanding as it requires populationwide data in order to separately identify the two types of fixed effects ${ }^{1}$. For this purpose, we use a unique data set covering the entire Danish population (5.5 mill. individuals) and all prescription drug purchases for a period of 11 years. Further, for each prescription redeemed, we observe IDs of both the patient and the doctor, which allow us to study the relative importance of

\footnotetext{
${ }^{1}$ The main concern is that the sample needs to be connected. See Woodcock (2005) for a discussion on how to potentially sample connected histories.
} 
both patient and doctor characteristics in explaining the variation of brand drug use. We study 7 blockbuster drugs that went off-patent ${ }^{2}$ in the period 2001 to 2005, e.g. Zocor and Losec.

We show that the propensity to prescribe brand drugs varies considerably over doctors/clinics with the bottom $5 \%$ prescribing brand-drugs on $27 \%$ of their prescriptions, compared to $81 \%$ for the top $95 \%$. We find that unobserved patient characteristics, such as preferences, explain around $28 \%$ of the variation in drug choice, whereas unobserved doctor characteristics explain $0.7 \%$ of the variation in drug choice. Furthermore, observed patient and doctor characteristics are very poor predictors of drug choice, still, the choice of brand-name versions is positively correlated with both doctor and patient income and age of the prescribing doctor.

The results of this paper are obtained within an institutional framework where physicians do not have monetary incentives in the prescribing decision. Under this regime we find that the choice of more expensive brand-name drugs is almost entirely patient driven (relative to doctors). This is suggestive evidence that unless incentives schemes for doctors to sell brand drugs or more expensive drugs exist then doctors to not push these items.

The paper is organized as follows. Section 2 reviews the existing literature, section 3 describes the institutional setting, section 4 presents the data, section 5 provides the empirical methods followed by the results in section 6 . Section 7 concludes.

\footnotetext{
${ }^{2}$ As can be seen later, drugs still under patent protection exhibit market shares that are strictly less than one before the patent expires. This can be due to different factors, for example parallel import of brand drugs sold under different names. In other cases, patents are not granted exclusively to the given chemical substance, but also to how the substance is to be released in the human body. Companies producing generics can in some cases come up with alternative formulations that are close to the original brand medication.
} 


\section{Related literature}

When drugs go off-patent, the average price of treatment with the chemical compound often falls significantly. The patent for Zocor expired in Denmark in 2002, and within one year, the price of a day's supply of the drug fell almost $90 \%^{3}$. Although the efficacy of generics is believed to be on pair with that of brand drugs (see e.g. Kesselheim et al. (2008)), the prices of brand drugs often remain at the pre-patent level - or they may even go up. One strand of the literature on generic drug use focuses on the so-called 'generic competition paradox' coined after Scherer (1993). The paradox is based on the observation that the price of the branded version of a drug increases when the patent expires (Regan (2008)). One explanation for this paradox, as offered by Frank and Salkever (1992), is that the price-insensitive brand-loyal customers stick to the brand-name drug, losing the price sensitive segment to the generics.

Others, including Coscelli (2000) and Hellerstein (1998) argue that state dependence on both the patient and physician side can account for brand-versions to have positive market shares. With focus on the anti-ulcer market, Coscelli (2000) uses a $10 \%$ random sample of citizens aged 1585 in the Rome-area and finds a considerable degree of state dependence in the drug choice (generic vs. brand) for both patients and doctors. Hellerstein (1998) uses survey data on physicians and their patients in the US to examine the factors related to generic substitution. She finds considerable variation in the likelihood of prescribing generics over physicians, and that physicians are the key decision makers in the prescribing decision. However, why some physicians are more likely to prescribe the branded version of a drug is left unexplained.

\footnotetext{
${ }^{3}$ See http://infolink2003.elbo.dk/apotekerforeningen/dokumenter/doc/8000.pdf (in Danish).
} 
Using data on a sample of pharmacies in a Midwestern state in the US, Mott \& Cline (2002) use random effects logistic regressions to analyze characteristics associated with generic substitution. They find that unobserved doctor characteristics account for $23 \%$ of the variance in the opportunity for generic drug use. Similarly, unobserved characteristics of pharmacists accounted for $44 \%$ of the variance in the occurrence of generic substitution.

Granlund (2009) uses register data from the Swedish county of Västerbotten to investigate whether private sector physicians are more likely to veto generic substitution relative to publicly employed physicians. He finds that private physicians are 50-80 \% more likely to veto generic substitution and that the probability of the doctor vetoing generic substitution is increasing in the patients' co-payment. Also using Swedish data, Lundin (2010) finds that doctors in Sweden internalize the out of pocket price in the brand/generic decision.

More recently, lizuka (2012) uses micro data from Japan to show that doctors react to financial incentives in the prescribing decision, favoring brand drugs when this is financially attractive.

This paper adds to the literature by allowing for both patient and doctor fixed effects simultaneously (in contrast to e.g. Mott \& Cline (2002)). Our estimation strategy is tractable in the sense that both effects can have arbitrary correlation with observables with no distributional assumptions made about them. Further, we are, to our knowledge, the first to study this question using panel micro data for an entire nation. Besides being more representative compared to other data sets used in the literature, it also the technical advantage that observing all patients and all doctors allows us to identify all switches, which is what provides identification of the fixed effects. We do not offer any economic model for drug choice per se, but we think of the fixed effects as capturing - among other things - drug preferences on both the patient and doctor side. 


\section{Institutional setting}

We now provide an overview of the institutional setting relevant for the study. Denmark has universal and tax financed health insurance run by the government. This includes paid hospital treatments and GP visits. Prescription drug coverage is also part of the public health insurance plan, though with substantial co-payments. Co-payments are a function of yearly accumulated expenditures; consumers have to pay the full cost of prescription drugs if the yearly expenditures are below DKK 500, i.e., a DKK 500 deductible ${ }^{4}$. When reaching the DKK 500, co-payments are reduced to $50 \%$. Reaching DKK 1,200, co-payments will reduce to $25 \%$, and $15 \%$ at DKK 2,800. After exactly one year of initial purchase, the individual account is zeroed. By far the most, but not all prescription drugs, are subject to this general subsidy scheme ${ }^{5}$. Expenditures and co-payments are calculated on the basis of a reference pricing scheme. For drugs still under patent protection, the above co-payments will apply to the full price of a given product ${ }^{6}$. For offpatent drugs however, subsidies are only given on the basis of the cheapest generic substitute. This clearly gives the consumer an incentive to move to generic alternatives once a patent expires.

The Danish market for outpatient prescription drugs is highly regulated to secure correct handling of - as well as uniform prices on - drugs across pharmacies. Pharmaceutical companies report pharmacy purchase prices to the Danish Medicines Agency, who then announces retail prices. These retail prices (along with a comprehensive list of information about the specific drugs including generic substitutes ${ }^{7}$ ) are made publicly available and registered online for five

\footnotetext{
${ }^{4}$ DKK 500 is approximately US $\$ 100$.

${ }^{5}$ Other types of subsidies exist but none of them are crucial for this paper.

${ }^{6}$ Before April $1{ }^{\text {st }} 2005$, the reference price was calculated on the basis of the two cheapest generics.

${ }^{7}$ Substitutes are defined by having the same dose of the active substance as well as the same use (tablets, capsules etc).
} 
years. Furthermore, pharmaceutical companies must report changes in purchase prices two weeks in advance. Direct-to-consumer advertising for prescription drugs is illegal in Denmark, but it is legal for the drug companies to advertise to doctors.

Typically, the physician writes out a prescription containing dosage information and the brand version of the drug. When entering the pharmacy, the pharmacists are required by law ${ }^{8}$ to inform the patient of the existence of any generic alternatives to the brand version of the drug and how these compare in price and efficacy. The patient can then choose between the two (or more, if multiple generics are available). Doctors do not have monetary incentives to prescribe brand drugs over generics. They can mark on the prescription, if substitution is not to be allowed. We do not observe in the data whether this option has been filled. What we observe in the data is the brand information of the actual drug that the patient purchases. If some doctors systematically veto generic substitution, we will capture this in the physician fixed effect. In this sense, we do not discriminate between substitution being vetoed or sticking to the branded version being recommended verbally.

Table 1 lists the 7 drugs studied and their indication of use. They are used in the treatment of a variety of conditions, including depression, hypertension, epilepsy, hypercholesterolemia etc.

TABLE 1

SELECTED PATENT EXPIRIES DURING 2001-2005

\begin{tabular}{llll}
\hline \hline Brand-name & Chemical compund & ATC & Description \\
\hline Cipramil & citalopram & N06AB04 & Antidepressant \\
Losec & omeprazole & A02BC01 & Ulceration \\
Zocor & simvastatin & C10AA01 & Lipid lowering \\
Norvasc & amlodipine & C08CA01 & Antihypertension \\
Lamictal & lamotrigine & N03AX09 & Epilepsy \\
Imigran & sumatriptan & N02CC01 & Migrane \\
Selo-Zok & metoprolol & C07AB02 & Antihypertension \\
\hline
\end{tabular}

${ }^{8}$ This is also known as a generic substitution law. 
As in many other countries, a private insurance market exists. Relevant for the period of this study, only one company, 'danmark', offered insurance policies with additional coverage of prescription drug expenditures. Approximately $33 \%$ of the adult population is enrolled in 'danmark'. The requirements to be met when one wishes to obtain additional coverage through 'danmark' are strict: for example, it is not possible to enroll if one has purchased any prescription drugs during the preceding 12 months, and individuals above the age of 61 cannot enroll either. We do not observe whether people have additional private insurance, and the effect of additional private insurance on drug choice will be captured in the individual specific effect. Given the criteria of enrollment, it is not unreasonable to think of this as a part of the fixed effect.

\section{Data}

We use administrative data provided by Statistics Denmark. The data set contains information on all Danish individuals above the age of 18 in the period from 1998-2008. For each individual in this period, we know the complete history of prescription drug purchases including date, price, subsidies, type of drug, and trade name of the drug. These data are augmented with socioeconomic information describing demographics, such as income and age.

In the prescription data, we observe an ID of the prescribing clinic. Ideally, we would like to have physician specific IDs, but this is not possible. However, we observe the identity of the doctors working under a given clinic ID at any given point in time. We can then identify these doctors in the demographics data set as well. For clinics with more than one doctor, we can calculate average income and age etc. Out of the 2,817 clinics in the data, around $66 \%$ are oneman clinics, $20 \%$ have two doctors, $8 \%$ have three doctors, $4 \%$ have four doctors, and less than 
$3 \%$ have 5 or more doctors. We have performed sensitivity checks of the main results based on clinics with only one doctor versus clinics with more doctors. The results are not sensitive. In the following we use the words clinic and doctors interchangeably ${ }^{9}$.

Figure 1 shows the market share of brands over time for the drugs studied. Clearly there is a drug specific component as to how brand marked shares evolve over time. For example, the branded drug Lamictal goes from a market share of (almost) $100 \%$ to one below $30 \%$ over a period of just 3 months. On the other hand, a drug like Selo-Zok maintains a market share above $30 \%$ for the entire data period.

\section{FIGURE 1}

BRAND MARKET SHARE - BY DRUG

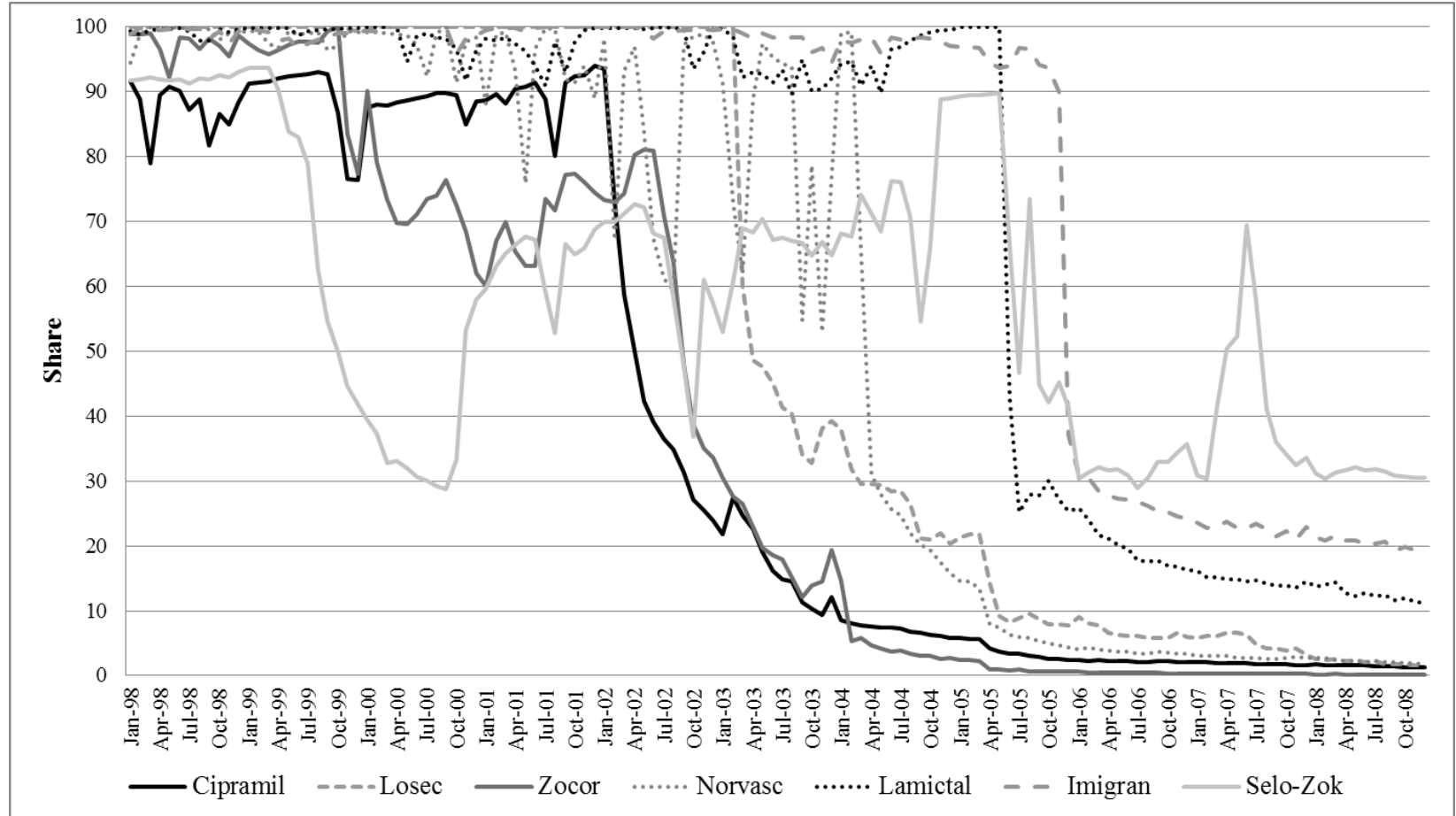

Note: Some drugs' brand shares are strictly less than one over the entire data period. This is mainly due to parallel imports, which is branded versions sold under different names. The drug Zelo-Zok exhibits a peculiar pattern with a drastically falling market share from late 1999/early 2000, after which it increases again. This is caused by a generic entrant that challenges the brand-firm's patent. The generic entrant was forced to withdraw from the market and hence the brand share increased again.

\footnotetext{
${ }^{9}$ Results are available upon request.
} 
FIGURE 2

Relative DRUG PRICE - BY DRUg GROUP

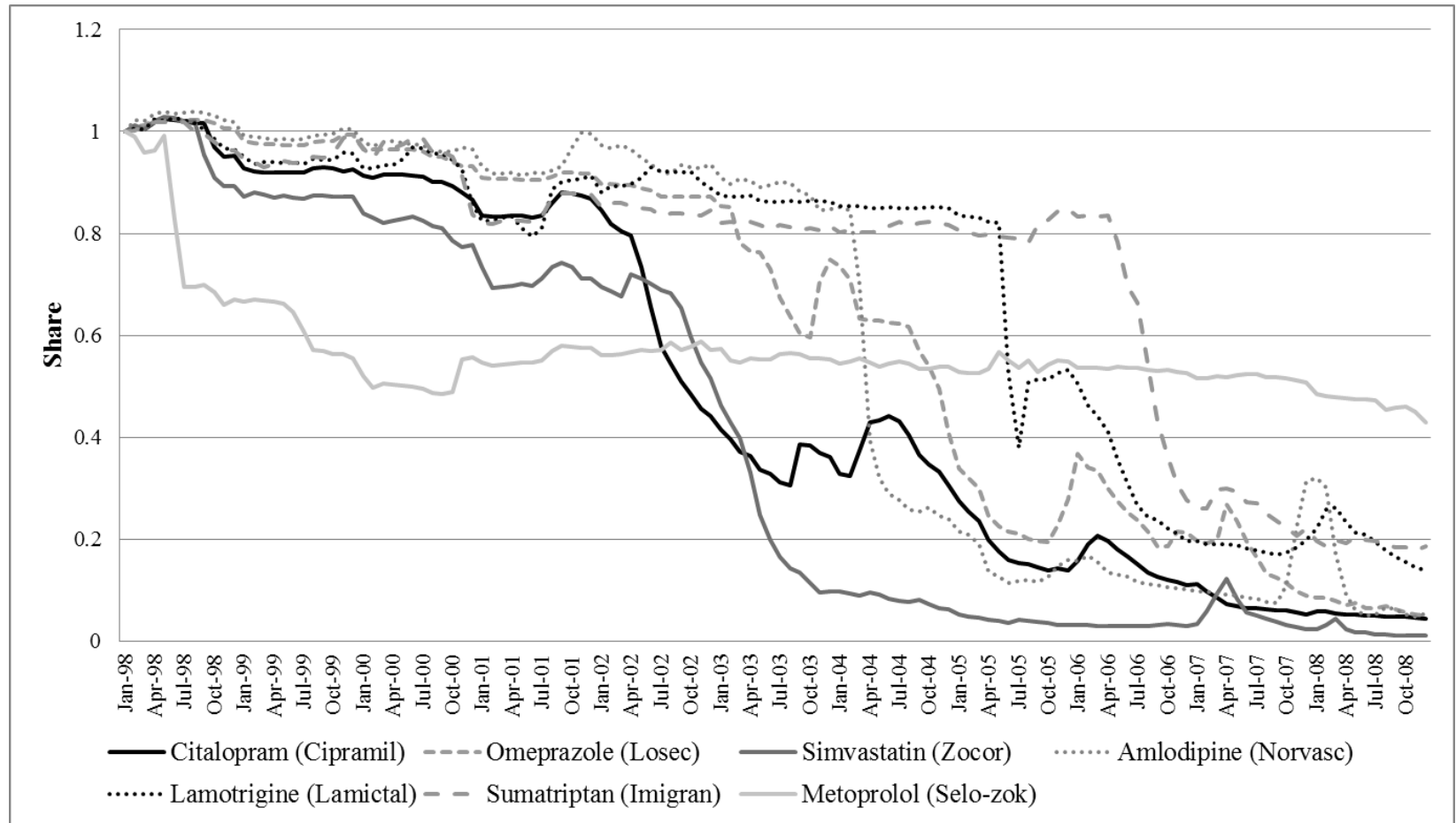

Note: The average price of a defined daily dose is calculated on the drug group-month level. These are then taken relative to the price as of January 1998. It is the average price of the entire drug group, so both generics and brands are included.

In figure 2 , the relative price of the drug groups are plotted over time. The relative price falls markedly in most drug groups, typically with prices down to only a couple of percent relative to pre-patent periods. For estimation purposes, we control for the above changes in prices by including detailed drug specific time effects (quarterly). This should capture the above price effects as well as general time trends. We believe this to be the most flexible way to deal with price variations. As indicated by figure 1 and 2 much of the variation explained by the drugquarter dummies will be capturing price changes.

To make sure that we have some variation in the outcome, we only include observations in the final data set where the month-drug market share is between 5 and $95 \%$. This is done since the linear probability model that we use does not capture well extreme cases, such as periods where 
all doctors prescribe only the brand drug ${ }^{10}$. This reduces the number of prescriptions from $25,820,892$ to $13,415,012$, purchased by 957,869 different individuals, written out by 2,817 different clinics. The mean brand share and total number of prescriptions by drug type for the estimation sample can be found in table 2 .

TABLE 2

DESCRIPTIVE STATISTICS ON PRESCRIPTIONS

\begin{tabular}{|c|c|c|c|c|}
\hline $\begin{array}{l}\text { Chemical } \\
\text { compound }\end{array}$ & $\begin{array}{l}\text { Brand } \\
\text { version }\end{array}$ & \# Prescriptions & $\begin{array}{l}\text { Mean brand } \\
\text { share }\end{array}$ & $\begin{array}{l}\text { Standard } \\
\text { Deviation }\end{array}$ \\
\hline Omeprazole & Losec & 647,028 & 0.19 & 0.39 \\
\hline Metoprolol & Selo-Zok & $5,822,235$ & 0.52 & 0.50 \\
\hline Amlodipine & Norvasc & $1,622,957$ & 0.51 & 0.50 \\
\hline Simvastatin & Zocor & $1,149,526$ & 0.45 & 0.50 \\
\hline Sumatriptan & Imigran & 586,972 & 0.34 & 0.47 \\
\hline Lamotrigine & Lamictal & 597,864 & 0.34 & 0.47 \\
\hline Citalopram & Cipramil & $2,988,430$ & 0.50 & 0.50 \\
\hline \multicolumn{2}{|c|}{ Total } & $13,415,012$ & 0.48 & 0.50 \\
\hline
\end{tabular}

Along with information on the prescriptions redeemed, we also observe information on the individuals who redeem them. As we are interested in estimating models with fixed effects, we only include time varying covariates. Table 3 reports descriptive statistics on the patients and doctors in the sample. The age of the average person in the sample is 60.69 years, and $8 \%$ experience unemployment during the study period. The average patient picks up 3.39 prescriptions a year of the drugs studied. The number of doctors per clinic is around 1.6, and the average income per doctor is DKK 889,466.

\footnotetext{
${ }^{10}$ The results are not sensitive to this specification. We have tried different cut-off rules and the results do not change.
} 
TABLE 3

DESCRIPTIVE STATISTICS -

PATIENTS AND DOCTORS

\begin{tabular}{|c|c|c|}
\hline \multicolumn{3}{|l|}{ Patients } \\
\hline & Mean & Std. Dev. \\
\hline Income & $221,889.40$ & $210,522.70$ \\
\hline Age & 60.69 & 17.19 \\
\hline Avg. \# prescriptions/year & 3.39 & 2.67 \\
\hline Unemployment & 0.08 & 0.28 \\
\hline
\end{tabular}

Doctors

\begin{tabular}{l|rr} 
& Mean & Std. Dev. \\
\hline List size & 2,312 & $1,510.74$ \\
Doctors & 1.60 & 1.03 \\
Age & 53.13 & 6.62 \\
Income & $889,466.20$ & $410,947.20$ \\
\hline
\end{tabular}

\# Obs. $\quad 2,817$

Note: Income is measured in DKK (US\$ $1=$ DKK 6) in 2008 prices.

Unemployment is a dummy variable equal to 1 if the individual has been

unemployed. Share of brand prescriptions is the fraction of the individual's

total number of prescriptions that are brand-versions. This is calculated

conditional on having redeemed at least 2 prescriptions.

As we will discuss later, the doctor fixed effects are identified by the patients who switch doctors. Thus, it is extremely important that there are enough switchers in the sample. Around 24 $\%$ of the individuals in the sample see more than one doctor, amounting to 229,280 individuals; see table 4 .

TABLE 4

NUMBER OF DIFFERENT PHYSICANS VISITED BY PATIENTS

\begin{tabular}{l|ccccccccccccccc}
\hline \# Physicians visited & 1 & 2 & 3 & 4 & 5 & 6 & 7 & 8 & 9 & 10 & 11 & 12 & 13 & 14 & $15+$ \\
\hline Frequency & 728,859 & 160,920 & 45,754 & 14,173 & 4,819 & 1,775 & 778 & 318 & 170 & 99 & 52 & 40 & 29 & 20 & 63 \\
Percent & 76.09 & 16.80 & 4.78 & 1.48 & 0.50 & 0.19 & 0.08 & 0.03 & 0.02 & 0.01 & 0.01 & 0.00 & 0.00 & 0.00 & 0.01 \\
\hline
\end{tabular}

The mean brand shares of prescribed or picked up prescriptions for doctors and patients are reported in figure 3 . Some doctors seem to write out almost only brand versions. Thus, initially there seems initially to be a lot of variation in prescription tendencies by doctors. Hence, without 
patient information one might erroneously conclude that doctors matter a lot for the brand drug use. However, it might also be that specific doctors just have patients with a high tendency to demand brand drugs. The goal is to figure out if the variation is caused by doctors or patients.

\section{FIGURE 3}

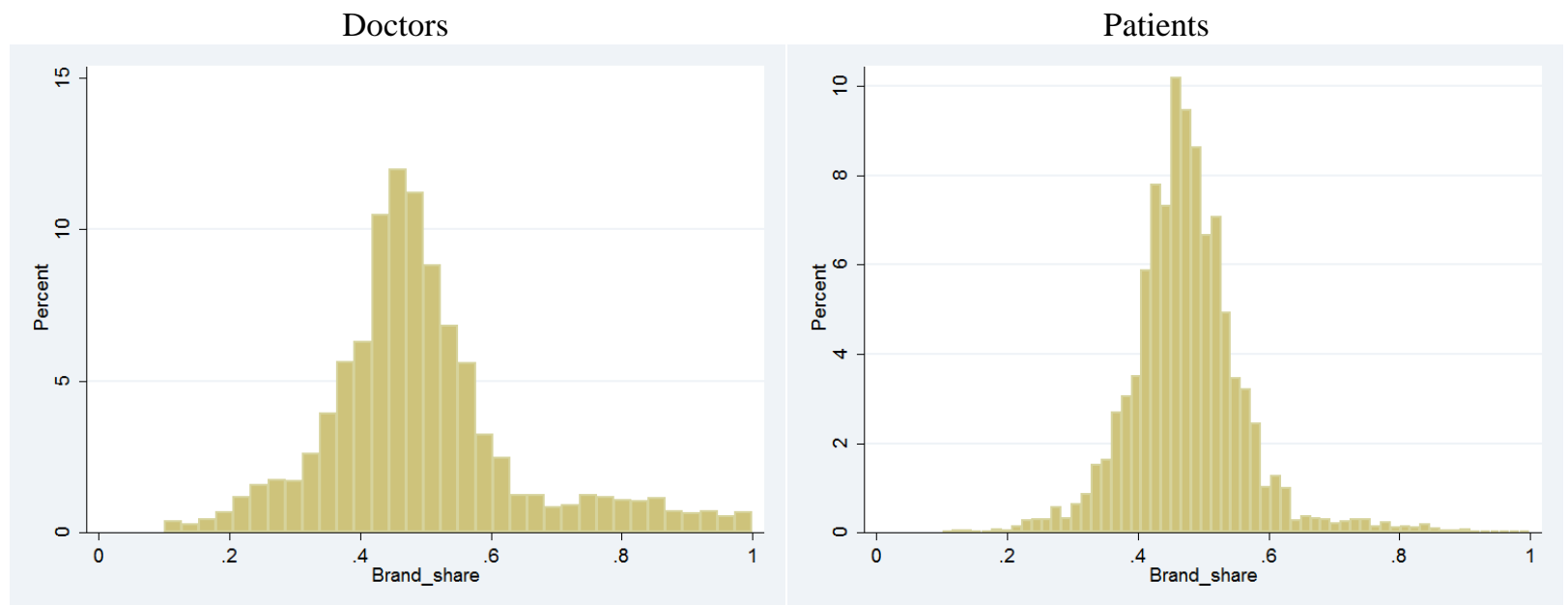

Note: Average shares of drugs purchased that are brands for doctors and patients. All years included.

\section{Empirical strategy}

For the main analysis we estimate four different linear probability models. We start out by estimating a standard (pooled) OLS model where the outcome is whether the redeemed prescription was brand or generic. Second, we estimate a model with patient fixed effects followed by a specification with doctor level fixed effects. The one-way fixed effects estimator used for two of the specifications is the standard within-group estimator that allows for the fixed effects to have arbitrarily correlation with the covariates. Lastly, we estimate a model that allows for both patient and doctor fixed effects simultaneously. In all four specifications, we include both patient and clinic level characteristics. An obvious explanatory variable to include here would be the price of the drug, or at least the price of the cheapest generic drug, given the effect 
the availability of generics has on prices. As we are not interested in estimating price elasticities per se, we think that the most flexible way to take these price effects into account is to include drug specific time dummies (quarterly level). We believe this is sufficient to capture the price variation we observe after generic entry.

\subsection{Econometric model}

Let $i \in\{1, \ldots, I\}$ index patients. Patient $i$ is represented by $N_{i}$ observations, so the total number of observations in the data is given by $N=\sum_{i=1}^{N} N_{i}$. The notation and the estimator thus allow for unbalanced and incomplete panels. The set of physicians is given by $\mathrm{j}=\{1, \ldots, \mathrm{J}\}$. We assume that patient $i$ 's $t^{\prime}$ th drug purchase $D_{i t}$ arises from the linear probability model:

$$
\mathrm{D}_{\mathrm{ijt}}=x_{i t} \beta_{x}+z_{i j} \beta_{z}+\theta_{\mathrm{i}}+\psi_{\mathrm{j}}+\sum_{t=1}^{T} Q_{t}+\varepsilon_{i t}
$$

where $\mathrm{x}_{\mathrm{it}}$ is a vector of observed patient time-varying covariates, $\mathrm{z}_{\mathrm{jt}}$ is a vector of observed physician time-varying covariates, $\theta_{\mathrm{i}}$ is a time-invariant patient effect capturing both observed and unobserved time-invariant characteristics of the patient, $\psi_{\mathrm{j}}$ is a time-invariant physician effect capturing both observed and unobserved time-invariant characteristics of the physician, $Q_{t}$ are quarterly dummies, and $\varepsilon_{i t}$ is the residual component.

We shall treat the residual $\varepsilon_{i t}$ as a genuine statistical residual. Hence, we impose the (identifying) assumption $E\left[\varepsilon_{i t} \mid x_{i t}, z_{j t}, \theta_{i}, \psi_{j}, Q_{t}\right\rfloor=0$. This assumption carries economic content in the sense that it rules out endogenous mobility. In other words, the assignment of physicians to 
patients is assumed independent of the residual component. We do not regard this assumption to be too restrictive since we suspect that many doctor switches are caused by a residential reallocation of patients.

In order to identify the full model we need a connected group of patients and physicians. This simply means that all patients and physicians have to be connected by other physicians or patients. A thorough discussion of identification of the model is presented in Abowd et al. (2002). It is impossible to compare the value of the estimated fixed effects between the nonconnected groups. Thus, we only use the largest connected group. Fortunately, this contains 99.99 percent of all the observations, which is caused by the many switchers as documented in Table 4.

The goal of the paper is to investigate if brand drug purchases are caused by patients or doctors. Hence, we are ultimately interested in the explanatory power of the observable patient and doctor characteristics, as well as the fixed effects and the drug specific time effects (that among other things capture price effects). For this purpose, we do a linear decomposition of the variation in the outcome. This is a further advantage of working with a linear model compared to a nonlinear model where a linear decomposition would not exist. The linear decomposition is fairly straight forward:

$$
\begin{aligned}
& \mathrm{V}\left(\mathrm{D}_{\mathrm{ijt}}\right)=\operatorname{Cov}\left(D_{i j t}, x_{i t} \beta_{x}+z_{i j} \beta_{z}+\theta_{\mathrm{i}}+\psi_{\mathrm{j}}+\varepsilon_{i t}\right) \\
& =\operatorname{Cov}\left(D_{i j t}, x_{i t} \beta_{x}\right)+\operatorname{Cov}\left(D_{i j t}, z_{i j} \beta_{z}\right)+\operatorname{Cov}\left(D_{i j t}, \theta_{\mathrm{i}}\right)+\operatorname{Cov}\left(D_{i j t}, \psi_{\mathrm{j}}\right)+\operatorname{Cov}\left(D_{i j t}, Q_{\mathrm{t}}\right)+\operatorname{Cov}\left(D_{i j t}, \varepsilon_{i t}\right)
\end{aligned}
$$

Dividing both sides by the variance, $\mathrm{V}\left(\mathrm{D}_{\mathrm{ij} \mathrm{t}}\right)$, we get a linear decomposition of the variance. That is, we will be able to see what the primary determinants that explain the variation in brand drug purchases are. 


\section{Results}

The results for the four different specifications are found in table 5. All specifications include detailed drug and drug-specific time controls. We emphasize that any interpretation of the estimated coefficients is purely correlation. Column 1 reports the OLS estimates ${ }^{11}$. Here there is no clear evidence on the relationship between income and choice of brand drugs. People who experience unemployment have a significantly lower probability of picking up a brand version, but the estimated effect is rather small corresponding to a $2.9 \%$ lower probability than those who are employed. There is a positive gradient in the doctors' income. R-square is around 0.33. This is mainly due to the drug/time effects - the socio-economic characteristics have very low explanatory power ${ }^{12}$.

Column 2 shows the results where patient level fixed effects are included. The evidence here is mixed compared to the results without any fixed effects. Now there seems to be a positive patient income gradient. Note however, that the source of variation used here is different due to the fixed effects (we now use within individual variation in contrast to column 1, where both within- and across individual variation is used). There is no longer clear evidence on association between doctor income and the outcome. Inclusion of patient fixed effects boosts the r-square by a factor of two.

\footnotetext{
${ }^{11}$ As always, researchers should take care using OLS when considering binary outcomes. We have calculated the predictions from the full model and plotted them. These can be found in figure A1 in appendix A. Around $70 \%$ of our predicted values fall within the $0 / 1$ interval.

${ }^{12}$ We have not included age in the OLS regression. The reason is that in the full model the effect of age cannot be estimated, since including time dummies, patient fixed effect, and age would cause multicollinearity. In the OLS, regression age has a positive effect on choosing a brand drug.
} 
Turning to the model with doctor fixed effects in column 3, patient income is now negatively correlated with choosing a brand-name. Doctor's income also seems positively related to brand choice. We see that the inclusion of doctor fixed effects only increases r-square by 0.01 relative to the OLS without any fixed effects. This is the first indication that doctors do not push brandname drugs.

The full model that allows for both types of fixed effects are presented in column 4 . Note that no standard errors are reported due to computational limitations. Here we see a positive income gradient on the patient side. Here, the more affluent doctors seem to be those who prescribe brand drugs. We estimate the correlation between the patient and doctor fixed effect to be -.03. Thus, there seems to a small negative sorting between the fixed effects. This might seem counter intuitive as it suggests that patients with a high preference for brand drugs have doctors that are reluctant to prescribe them. However, the correlation is small and suffers from bias. We will get back to this issue in the robustness section. We have conducted F-tests for the joint significance of the fixed effects. For the full model, this is also done separately for the doctor- and patient effects. The effects are significantly different from zero using all conventional levels of significance. 
TABLE $5^{\mathrm{a}}$

ESTIMATION RESULTS FOR THE FOUR DIFFERENT SPECIFICATIONS

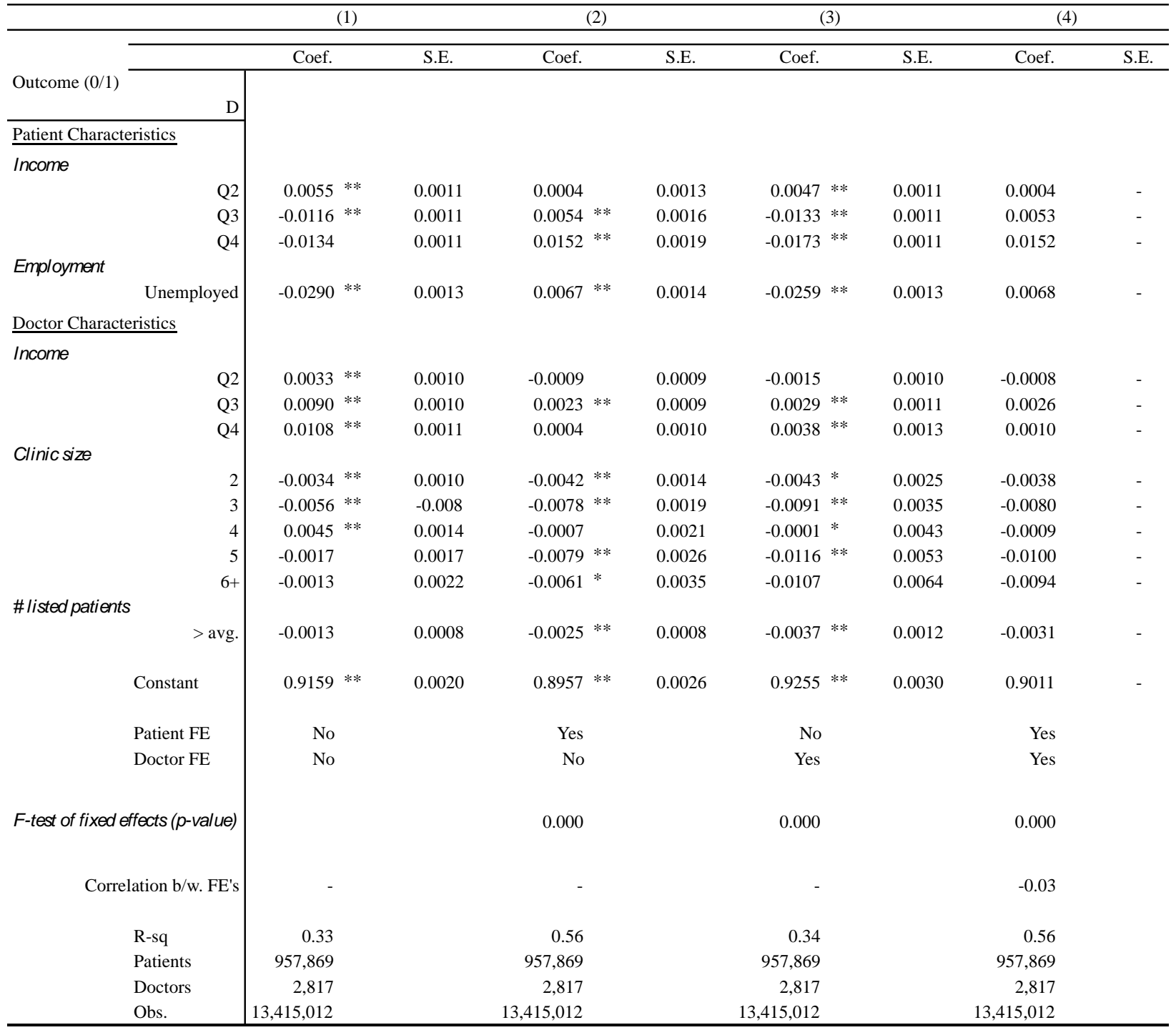

a ** indicates significance at the 5\% level. All covariates enter as dummy variables. 'Coef.' is the coefficient estimate, and S.E. is the associated standard error. Standard errors are not reported for the full model including two-way fixed effects. These are only available by bootstrapping which is infeasible.

The linear variance decompositions from the four different models are found in table 6 . In specification 1 , both patient and doctor observable characteristics explain less than 0.00 percent of the variation in the outcome. The time/drug specific factors explain $32.7 \%$ of the variation. The patient fixed effects explain $28.8 \%$ of the variation in the outcome; see column 2 in table 6. When we include the doctor fixed effects instead, these pick up only $1.5 \%$ of the variation. For 
the full model in column 4, patient fixed effects explain $26.8 \%$ of the variation, doctor fixed effects explain $0.7 \%$ of the variation, and the drug/time specific effects explain $31.3 \%{ }^{13}$.

TABLE 6

\begin{tabular}{|c|c|c|c|c|}
\hline & (1) & $(2)$ & (3) & (4) \\
\hline Total & 1.00 & 1.00 & 1.00 & 1.00 \\
\hline Patient Effect & - & $28.8 \%$ & - & $26.8 \%$ \\
\hline Doctor Effect & - & - & $1.5 \%$ & $0.7 \%$ \\
\hline Patient char. & $0.0 \%$ & $0.0 \%$ & $0.0 \%$ & $-0.1 \%$ \\
\hline Doctor char. & $0.0 \%$ & $0.0 \%$ & $0.0 \%$ & $0.0 \%$ \\
\hline Time/drug char. & $32.7 \%$ & $31.3 \%$ & $32.7 \%$ & $31.3 \%$ \\
\hline Residual & $67.3 \%$ & $41.2 \%$ & $65.8 \%$ & $41.2 \%$ \\
\hline
\end{tabular}

A potential worry is that the high explanatory power of the patient is spurious. If a patient has only one observation, then the patient fixed effect will perfectly explain the residual variation. Basically, the worry is that the patient fixed effect is picking up the residual making the fit 'too well'. To look into this, we do the above decomposition (for the full model) by the number of prescriptions per individual, i.e., on the $\mathrm{x}$-axis we keep only patients that have 1,2 , 3 , etc. prescriptions and then plot the variance decomposition for these individuals. This is illustrated in figure 4. As expected, the explanatory power of the patient specific effect is higher for individuals who are only observed with one or two prescriptions (around $50 \%$ of the variation). As we perform the variance decomposition for patients who have 10 or 15 prescriptions, the relationship looks stable with the patient effect explaining just below $20 \%$ of the variation, which is close to what we found using all prescriptions.

\footnotetext{
13 A possible concern here is that we have more doctors in some clinics and that this may somehow blur the importance of the clinic fixed effects. To shed light on this, the variance decomposition has been done using only one-man clinics. The results are identical to that of the full sample (available from the author upon request).
} 
FIGURE 4

VARIANCE DECOMPOSITION BY NUMBER OF PRESCRIPTIONS PER PATIENT

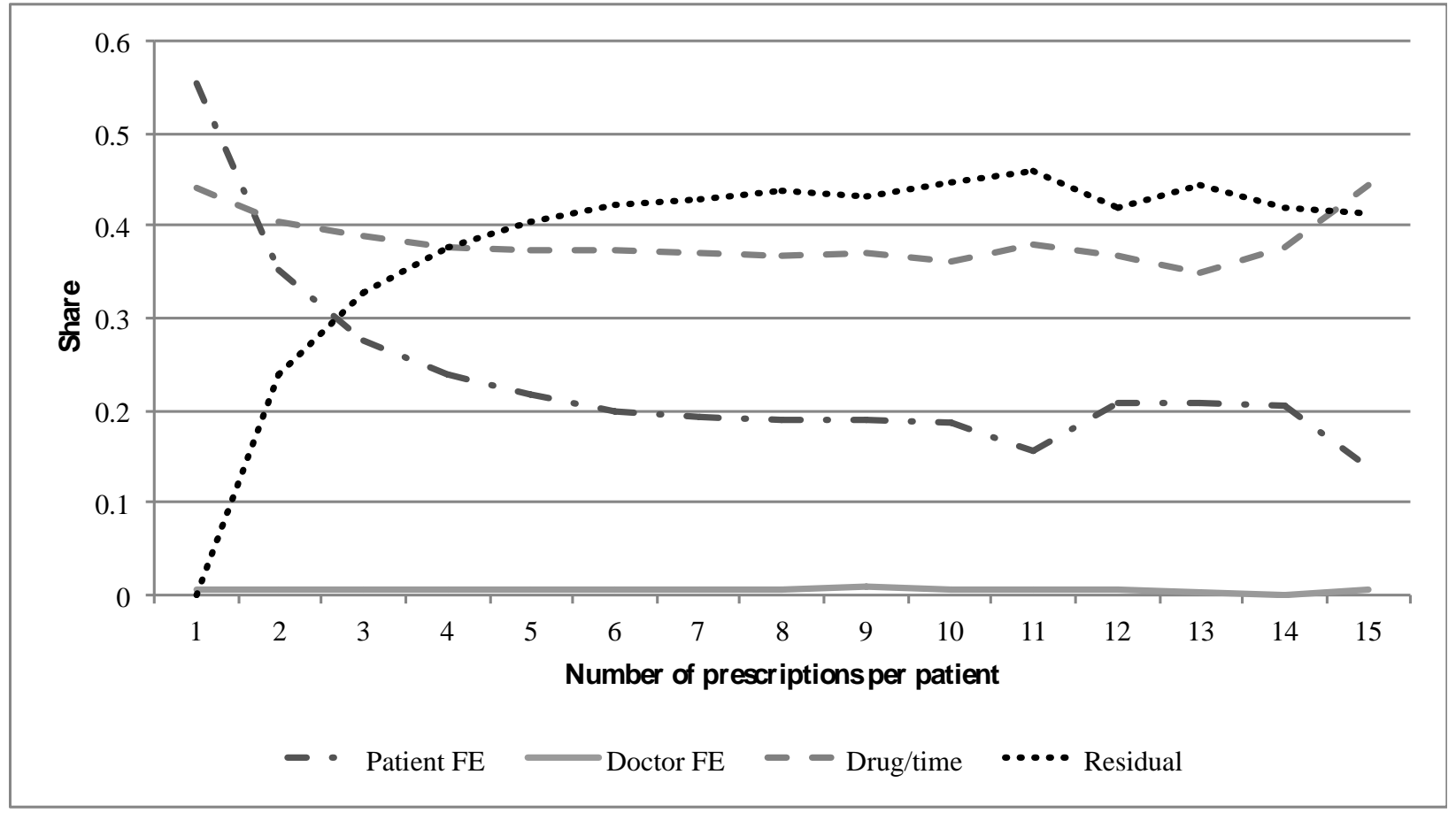

Variance decomposition by the number of prescriptions redeemed by individuals.

One might think of the decision to stick with the brand medication as a static doctor-patient-drug pair specific outcome and that retaining multiple observations on this level would be misleading. We have estimated the full model where we have only included unique doctor-patient-drug matches (this reduces the sample to $12 \%$ of that of the original estimation sample). Doing this has the effect that a larger fraction of the variation is now explained by the person specific component (results are available from the authors on request).

\subsection{Small Samples}

The above model is in principle not estimable when researchers do not have access to population level data - which is what most of the existing literature has access to - since the largest connected group will be a small fraction of the sample, when the sample is small relative to the population. However, even estimating simple one-way fixed effects models can be troublesome 
if the number of fixed effects are growing non-linearly in the sample size. This is the case with small random samples of patients since these will contain a relatively large part of the physicians, i.e., for each additional patient sampled, the probability of sampling a new physician is declining in the sample size. This may lead the researcher to overestimate the importance of e.g. physician effects ${ }^{14}$. To show this, we estimate our three different specifications that include fixed effects and do the variance decomposition by varying sample size. We draw random samples of our data on the patient level. In the left panel of figure 5, we have plotted the contribution of doctor and patient fixed effects when these are estimated sequentially on smaller samples. We then plot their explanatory power from the variance decomposition as a function of the sample size. The smallest sample $(0.1 \%)$ includes 13,333 prescriptions, 952 patients, and 937 clinics. Using such a sample, one might erroneously conclude that physician fixed effects explain around $35 \%$ of the variation in drug choice ${ }^{15}$. An implication of this for policy would be that physicians should be targeted to push generic drug use. Increasing the sample size dramatically decreases the importance of the doctor fixed effects. In the left panel we have also plotted the contribution of the patient effects, and the relationship here is constant over the sample size. Note that the number of patients will be growing in sample size. In the right panel of figure 4, the same exercise is carried out, however the effects are estimated jointly. Here the explanatory power of the two effects is almost constant ${ }^{16}$.

\footnotetext{
${ }^{14}$ Mott \& Cline (2002) implement a variance decomposition similar to what is done in this paper estimating specifications with pharmacy and doctor fixed effects sequentially. They find that these fixed effects explain a large part of the variation in generic drug use. However, they have a sample with 6,380 prescriptions, written out by 3,934 prescribers and collected at 758 different pharmacies. Having only 1.6 as many observations (with doctor fixed effects) will artificially fit the data very well, leading to these fixed effects being good predictors of the variation in the data.

${ }^{15}$ There are many effects relative to the sample size, and they also pick up the patient fixed effects.

${ }^{16}$ The largest connected groups are small for the full model when we do the sampling scheme. For example, the largest connected group for the $0.1 \%$ sample consists of only 475 prescriptions.
} 
FIGURE $5^{\mathrm{a}}$

VARIANCE DECOMPOSITION BY SAMPLE SIZE

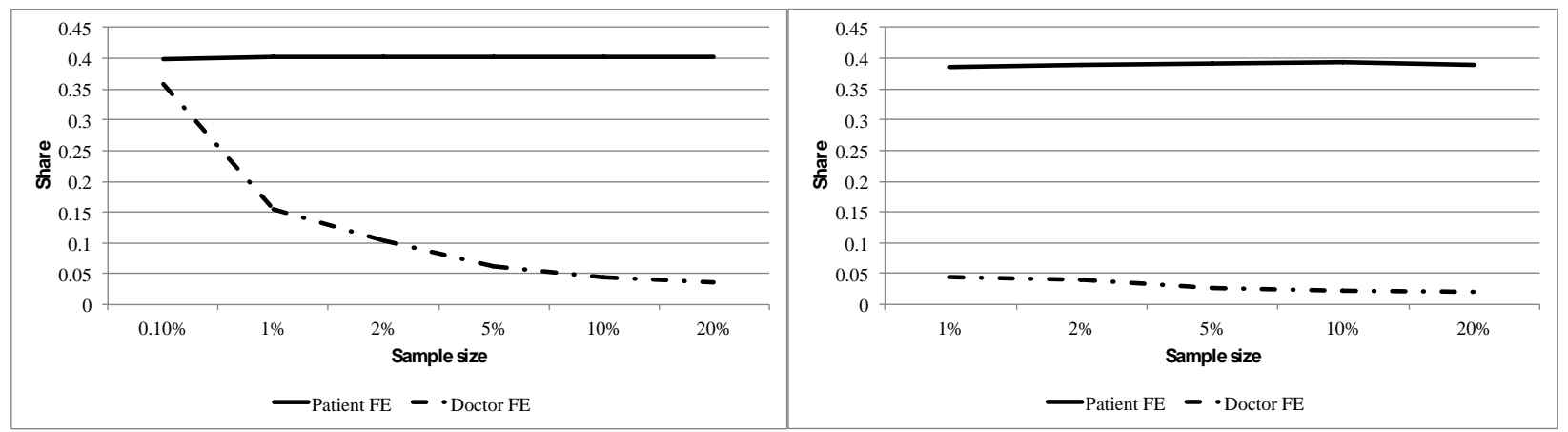

${ }^{\text {a }}$ Samples are drawn randomly on the patient level. The left panel shows the contribution to the variance from patient and doctor fixed effects obtained from one-way fixed effect models by varying sample size. The right panel shows the decomposition from the model where the effects are estimated jointly. The model that includes both fixed effects does not convey any meaningful results with a sample of $0.1 \%$ as the largest connected group consists of only 475 observations. Thus this is excluded.

The results suggest that one should be very careful when using a random sample combined with fixed effect estimators. Even relatively large random samples potentially overestimate the importance of the fixed effects.

\subsection{Robustness}

In this section we perform two robustness checks. The main worry is that the FE estimators are not consistent. The estimator is consistent if $N_{i} \rightarrow \infty$ for all $i$ holding the number of doctors constant. This is clearly not the case, and therefore the fixed effects estimated will suffer from standard estimation error.

In order to judge the size of the bias and the standard estimation error, we estimate the model without covariates on the real data (column 1 in Table 7$)^{17}$. We retain the allocation of patients to doctors as found in the data. We then simulate two versions of the model. First, we simulate a counterfactual individual outcome by independently and randomly sampling from a uniform

\footnotetext{
${ }^{17}$ The exercise could be performed with covariates, but the bias has little to do with this, so we ignore them for expositional purposes.
} 
distribution on the interval from zero to one. If say $q$ percent of the picked up prescriptions are generic, we allocate $\mathrm{D}_{\mathrm{ijt}}=1$ if the random draw is above 1- $q$ and vice versa. We then estimate the model using the data structure and the generated outcome. The results are reported in column (2) of Table 7. Secondly, we draw patient and doctor FEs from the empirical marginal distributions of patient and doctor fixed effects, and the residual component as estimated in column (1) of Table 7. It generates a true zero correlation between patient and doctor fixed effect, but it now more resembles the true model. Again, we estimate the model on the data structure and the generated outcome and report the results in column (3) of Table 7.

One concern is that we only pick up the error term and that the estimated fixed effects therefore just reflect estimation error. Looking at column 2 in Table 7, we find that this is not the case. If the patient and doctor fixed effects only picked up random variation, then we would expect these to explain just as much of the variation in specification (2) as in (1). This is clearly not the case. Notice that the patient fixed effect still pick up some random errors since it artificially explains $7.2 \%$ of the variation in the outcome. However, this is far from the $38.7 \%$ that it explains in the true estimated model. Finally, F-tests testing the three hypotheses that patient fixed effects are jointly zero, that doctor fixed effects are jointly zero, and that both are jointly zero are all rejected at the 5 percent level. Thus, we conclude that the patient and doctor fixed effects in the model do not just pick up random variation from the error term.

Another concern is that the estimator for the correlation between the patient and the doctor FEs suffers from 'limited mobility bias' as discussed by for example Andrews et al. (2008). The intuition is that if a doctor fixed effect is under-estimated, e.g. due to standard estimation error, this doctor's patients will have overestimated patient effects. This generates a spurious negative correlation between the two fixed effects. In order to judge whether this is driving the negative 
correlation found in Table 5, we turn to column (3) in Table 7. Here we see that the correlation is estimated to be -.01, while we know that the true correlation was 0 , since this was the way the data was generated. In the true data the correlation was estimated to be -.12 (without covariates). We conclude that the bias in the correlation is of relatively little importance ${ }^{18}$. Thus, the small negative correlation found in Table 7 seems to be genuine and not the result of a large limited mobility bias in conjunction with a true positive correlation. However, notice that the correlation in Table 7 is small as well as negative, and notice that the negative correlation in column (2) of table 7 is caused by small standard deviations of the patient and doctor effects and not a large negative covariance.

TABLE 7

SENSITIVITY CHECKS

\begin{tabular}{|c|c|c|c|c|c|c|}
\hline Specification: & & $(1)$ & & (2) & & (3) \\
\hline \multicolumn{7}{|c|}{ Standard Deviation } \\
\hline Patient Effect & & 0.32 & & 0.14 & & 0.28 \\
\hline Doctor Effect & & 0.09 & & 0.02 & & 0.09 \\
\hline Correlation & & -0.12 & & -0.16 & & -0.01 \\
\hline \multicolumn{7}{|c|}{ F-test of fixed effects } \\
\hline Patient Effect & 8.30 & {$[0.00]$} & 1.00 & {$[0.06]$} & 6.39 & [0.00] \\
\hline Doctor Effect & 48.37 & {$[0.00]$} & 0.99 & {$[0.66]$} & 21.77 & [0.00] \\
\hline Both & 8.91 & {$[0.00]$} & 1.00 & {$[0.07]$} & 6.99 & [0.00] \\
\hline \multicolumn{7}{|c|}{ Variance Decomposition } \\
\hline Total & & $100 \%$ & & $100 \%$ & & $100 \%$ \\
\hline Patient Effect & & $38.7 \%$ & & $7.2 \%$ & & $32.1 \%$ \\
\hline Doctor Effect & & $2.0 \%$ & & $0.0 \%$ & & $2.9 \%$ \\
\hline Residual & & $59.3 \%$ & & $92.8 \%$ & & $65.0 \%$ \\
\hline Observations & & \multicolumn{5}{|c|}{$13,415,012$} \\
\hline
\end{tabular}

\footnotetext{
${ }^{18}$ This is also found in Bagger et al. (2013) using matched employer-employee data on wages.
} 


\section{Conclusion}

In the Danish context where physicians have no financial incentives in the prescribing decision, we find that time invariant unobserved clinic characteristics explain $0.7 \%$ of the variation in generic drug use for seven frequently utilized medications. This is in stark contrast to the study by Mott \& Cline (2002). We find that unobserved time invariant patient characteristics, including preferences, explain around $26.8 \%$ of the variation in generic drug purchases. Surprisingly, we find the socio-economic characteristics of both patients and doctors to have very low explanatory power. Even though prices are not modeled explicitly in this paper, the introduction of generics and their inherent impact on the price of treatment seem to explain roughly one third of the variation in drug choice.

We have presented evidence that the diffusion of generics (often) happens very quickly in Denmark. This is in contrast to the findings in Iizuka (2012) where this diffusion is shown to be markedly slower in the Japanese market. This is suggested by lizuka (2012) to be related to the fact that physicians in Japan have incentives to prescribe more expensive brand drugs. Taken together with our finding that physicians in Denmark do not seem to push brand drugs, this is suggestive evidence that removing incentives for physicians to promote brand drugs are important if one wishes to increase generic drug use. However in order to make such strong conclusions we would like an experiment where physicians face different incentives schemes. We will leave this for future research.

Finally, we provide suggestive evidence that using only a small random sample of patients can give very misleading results. In our setting this results in a grossly overestimated explanatory power of the doctor fixed effect that vanishes as the sample size gets bigger. 


\section{References}

Abowd, J. M., Kramarz, F. \& Margolis, D., N. (1999): High Wage Workers and High Wage Firms, Econometrica, Vol. 67, pp. 251-333.

Abowd, J.M., Creecy, R.H., Kramarz, F. (2002): Computing person and firm effects using linked longitudinal employer-employee data, Mimeo.

Andrews, M. J., Gill, L., Schank, T., Upward, R. (2008): High wage workers and low wage firms: negative assortative matching or limited mobility bias? Journal of the Royal Statistical Society, Vol. 171, 3, pp. 673-697

Bagger, J., Sørensen, K., Vejlin. R (2013): Wage Sorting Trends, Economics Letters, Vol. 118, 1, pp. 63-67

Bennett, Daniel, Che-Lun Hung, Tsai-Ling Lauderdale (2011), "Health Care Competition and Antibiotic Use in Taiwan", working paper, University of Chicago.

Coscelli, A. (2000): The Importance of Doctors' and Patients' Preferences in the Prescription Decision, The Journal of Industrial Economics, Vol. 48, pp. 349-369.

Frank, R. G. \& Salkever, D. S. (1997): Pricing Patent Loss and the Market for Pharmaceuticals, Southern Economic Journal, Vol. 59, pp. 165-179.

Granlund, D. (2009): Are Private Physicians More Likely to Veto Generic Substitution of Prescribed Pharmaceuticals?, Social Sciences \& Medicine, Vol. 69, pp. 1643-1650

Hellerstein, J. K. (1998): The Importance of the Physician in the Generic versus Trade-Name Prescription Decision, The RAND Journal of Economics, Vol. 29, pp. 108-136.

Iizuka, T. (2012): Physician Agency and Adoption of Generic Pharmaceuticals, American Economic Review, vol. 102, pp. 2826-2858.

Kesselheim, A. S., Misono, A. S., Lee, J. L., Stedman, M. R, Brookhart, M. A., Choudhry, N. K., Shrank, W. H. (2008): Clinical Equivalence of Generic and Brand-Name Drugs Used in Cardiovascular Disease, The Journal of the American Medical Association, Vol. 300, pp. 25142526.

Koulayev, S., Simeonova, E., Skipper, N.: Who is in Control? The Determinants of Patient Adherence with Medication Therapy,

Manuscript. http://mit.econ.au.dk/vip_htm/nskipper/adherence_desc.pdf

Lundin, D. (2000): Moral Hazard in the Physician Prescription Behavior, Journal of Health Economics, vol. 19, pp. 639-662.

Mott, D. A. \& Cline, R. R. (2002): Exploring Generic Drug Use Behavior. The Role of Prescribers and Pharmacists in the Opportunity for Generic Drug Use and Generic Substitution. Medical Care, Vol. 40, pp. 662-674. 
Regan, T. L. (2008): Generic Entry, Price Competition, and Market Segmentation in the Prescription Drug Market, International Journal of Industrial Organization, Vol. 26, pp. 930948.

Scherer, F. M. (1999): Pricing, profits, and technological progress in the pharmaceutical industry, Journal of Economic Perspectives, Vol., 7, pp. 97-115.

Woodcock, S. (2005): Sampling Connected Histories from Longitudinal Linked Data, Working Paper 
Appendix A

FIGURE A1

DISTRIBUTION OF PREDICTIONS FROM FULL MODEL

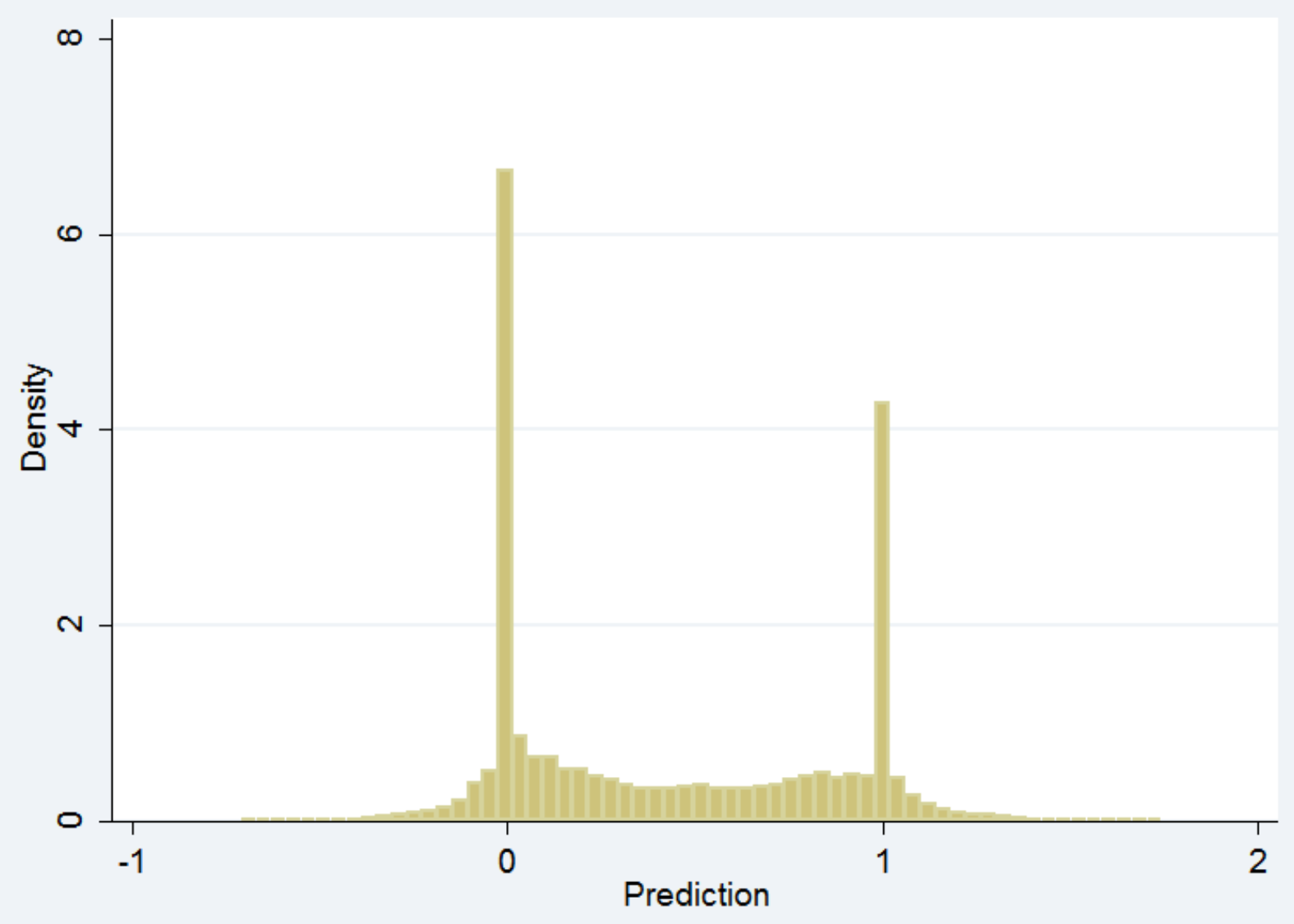




\section{Economics Working Papers}

2012-21: Christina Gravert: Can a sense of entitlement increase stealing?

2012-22: $\quad$ Sofie Kragh Pedersen, Alexander K. Koch and Julia Nafziger: Who wants paternalism?

2012-23: $\quad$ Astrid Würtz Rasmussen and Leslie S. Stratton: How Distance to a Non-Residential Parent Relates to Child Outcomes

2012-24: Nabanita Datta Gupta, Mette Lausten and Dario Pozzoli: Does Mother Know Best? Parental Discrepancies in Assessing Child Functioning

2012-25: $\quad$ Nabanita Datta Gupta, Daniel Lau and Dario Pozzoli: The Impact of Education and Occupation on Temporary and Permanent Work Incapacity

2012-26: Jonas Maibom Pedersen, Michael Rosholm and Michael Svarer: Experimental Evidence on the Effects of Early Meetings and Activation

2012-27: Pieter Gautier, Paul Muller, Bas van der Klaauw, Michael Rosholm and Michael Svarer: Estimating Equilibrium Effects of Job Search Assistance

2012-28: Elena Cottini and Paolo Ghinetti: Working Conditions, Lifestyles and Health

2012-29: $\quad$ Casper Worm Hansen: Causes of mortality and development: Evidence from large health shocks in 20th century America

2013-01: Juanna Schrøter Joensen and Helena Skyt Nielsen: Math and Gender: Is Math a Route to a High-Powered Career?

2013-02: Valerie Smeets, Michael Waldman and Frederic Warzynski: Performance, Career Dynamics, and Span of Control

2013-03: Rasmus Landersø, Helena Skyt Nielsen and Marianne Simonsen: School Starting Age and Crime

2013-04: Nabanita Datta Gupta and Lene Kromann: An Equilibrium Search Model of the Labor Market Entry of Second-Generation Immigrants and Ethnic Danes

2013-04: Niels Skipper and Rune Vejlin: Determinants of Generic vs. Brand Drug Choice: Evidence from Population-wide Danish Data 